\title{
RANK-DEPENDENT GALTON-WATSON PROCESSES AND THEIR PATHWISE DUALS
}

\author{
BY SERIK SAGITOV AND JONAS JAGERS
}

\begin{abstract}
We introduce a modified Galton-Watson process using the framework of an infinite system of particles labelled by $(x, t)$, where $x$ is the rank of the particle born at time $t$. The key assumption concerning the offspring numbers of different particles is that they are independent, but their distributions may depend on the particle label $(x, t)$. For the associated system of coupled monotone Markov chains, we address the issue of pathwise duality elucidated by a remarkable graphical representation in which the trajectories of the primary Markov chains and their duals coalesce to form forest graphs on a twodimensional grid.
\end{abstract}

Keywords: Graphical representation; Siegmund's duality; dual forest; linear-fractional reproduction; birth-death process

2010 Mathematics Subject Classification: Primary 60J80

Secondary $60 \mathrm{~J} 10$

\section{Introduction}

The Galton-Watson (GW) process is a basic stochastic model for the generation size in a population of reproducing particles (see, e.g. [3]). Slightly modifying the framework of [5], we define a GW process in terms of an infinite system of particles uniquely labelled by pairs $(x, t) \in \mathbb{N} \times \mathbb{Z}$, where $t$ refers to the generation number and $x$ is the rank of the particle within this generation. Given a set of independent and identically distributed (i.i.d.) $\mathbb{N}_{0}$-valued random variables (here, $\mathbb{N}_{0}=\{0\} \cup \mathbb{N}$ )

$$
\left\{u_{t}(x)\right\}_{(x, t) \in \mathbb{N} \times \mathbb{Z},}
$$

a GW process stemming from $Z_{a}$ particles at time $a \in \mathbb{Z}$ is the Markov chain $\left\{Z_{t}\right\}_{t \geq a}$ characterized by the branching property

$$
Z_{t+1}=\sum_{x=1}^{Z_{t}} u_{t}(x),
$$

where $u_{t}(x)$ represents the number of offspring of the particle $(x, t)$. Relation (2) induces the following rank-inheritance rules:

- each particle $(x, t+1)$ has a unique parent $\left(x^{\prime}, t\right)$, and

- if $x<y$, and $\left(x^{\prime}, t\right)$ and $\left(y^{\prime}, t\right)$ are the respective parents of $(x, t+1)$ and $(y, t+1)$, then $x^{\prime} \leq y^{\prime}$. 
For example, if $u_{t}(1)=k \geq 1$ then $k$ children of the rank-1 particle get the ranks $1, \ldots, k$ among the particles born at time $t+1$. The ranks of particles play no role in the standard GW setting; however, Bertoin [5] used them in studying GW processes with neutral mutations.

In this paper we introduce a new modification of the GW model by allowing the rank of a particle to determine its reproduction law. In a general rank-dependent GW setting, the independent offspring numbers $u_{t}(x)$ have distributions that vary over the birth times $t$ and particle ranks $x$. To illustrate, consider a linear-fractional reproduction law

$$
\mathbb{E}\left[s^{u_{t}(x)}\right]=1-q_{t}(x)+q_{t}(x) \frac{p_{t} s}{1-\left(1-p_{t}\right) s}, \quad p_{t} \in(0,1], q_{t}(x)=\mathbf{1}_{\{x \in\{1,3,5, \ldots\}\}},
$$

where the dependence on the particle rank takes effect via $q_{t}(x)$, the probability of having nonzero offspring. Here, particles with odd ranks always produce $k \geq 1$ offspring with probability $\left(1-p_{t}\right)^{k-1} p_{t}$, while particles of even ranks have no offspring. Note that in the time-homogeneous case with $p_{t} \equiv p$ and $q_{t}(x) \equiv q(x)$, the corresponding rank-dependent GW process cannot be treated as a two-type GW process, since the number of even-ranked children for the rank-3 particle depends on the number of children of the rank-1 particle.

The standard GW process has many extensions, usually motivated by biological applications (see [11] and [16]). Some of these extensions can be viewed as examples of rank-dependent GW processes: in Section 4 the scope of the rank-dependent GW setting is highlighted by referring to bounded GW processes, GW processes with immigration and emigration, duals to birth-death GW processes in a varying environment, as well as GW processes embedded in continuous-time linear birth-death processes in a varying environment. In particular, if the reproduction law $\mathrm{E}\left[s^{u_{t}(x)}\right]=f_{t}$ is not influenced by the particle rank, then the rank-dependent $\mathrm{GW}$ process is a $\mathrm{GW}$ process in a varying environment satisfying

$$
\mathbb{E}\left[s^{Z_{t}} \mid Z_{a}=z\right]=\left(f_{a} \circ \cdots \circ f_{t-1}(s)\right)^{z},
$$

where $f \circ g(s)$ denotes $f(g(s))$ (see [12], and also [4], [6], and [15], for recent treatments involving this model). In the rank-dependent GW setting, the last relation does not hold in general, making analysis more complicated.

The main results of the paper are collected in Section 3; proofs are given in Section 5. In Proposition 1 we consider the rank-dependent GW processes along with their pathwise dual processes, whose definition in Section 2 is based on Siegmund's duality; see [13], [27], and [28]. It shows, in particular, that the dual to the dual of a rank-dependent GW process is a shifted copy of the original rank-dependent GW process. In the literature on dual processes, the common setting involves time-homogeneous Markov processes. A notable exception is [2] treating a class of stationary processes. Our approach handles time-inhomogeneous Markov chains, and can even be adapted to the non-Markov setting, when, for example, the offspring number $u_{t}(x)$ depends on the offspring number $u_{t-1}\left(x^{\prime}\right)$ of the parent.

The infinite particle system framework permits an illuminating graphical representation of a system of coupled rank-dependent GW processes and their pathwise duals visualizing their trajectories as forest graphs. A process dual to an asexual reproduction model, like the GW process or Wright-Fisher model, is usually interpreted in terms of a coalescent model [10], [21]. Somewhat counterintuitively, our graphical representation indicates that the dual to a branching process is a form of branching process with dependencies (see Figure 1). The graphical representation also works for primary reproduction models with fixed population size, such as the Wright-Fisher model. 


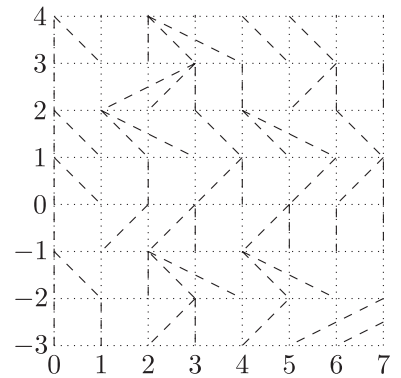

(a)

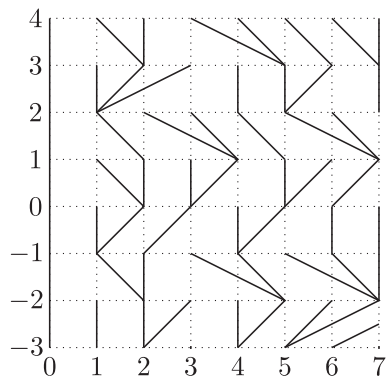

(b)

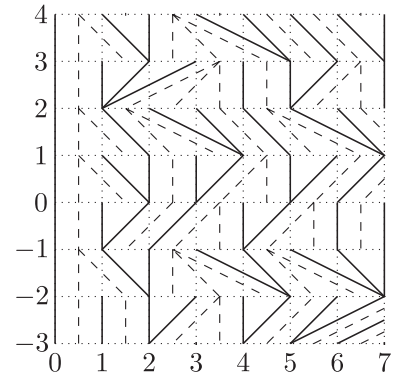

(c)

FIGURE 1: Graphical representations of the iterated reproduction mappings: (a) coupled trajectories of a rank-dependent GW process, (b) trajectories of the time-reversed process, (c) shifted trajectories from panel (a) do not cross with the trajectories from panel (b).

One of the examples in Section 4 shows that, even with a standard GW process, the dual Markov chain is not necessarily a rank-dependent GW process, because the dual offspring numbers become dependent on each other. An interesting open problem is to characterize the class of rank-dependent GW processes, whose dual Markov chain is itself a rank-dependent $\mathrm{GW}$ process. A simpler problem is to characterize the class of GW processes, whose dual Markov chain is itself a rank-dependent GW process.

We obtain two results addressing the latter problem. Considering the dual of the GW reproduction law, in Proposition 2 we state that the marginal dual offspring distribution is always linear fractional. In Theorem 1 we demonstrate that the dual process is GW with an eternal particle if and only if the primary reproduction law is itself linear fractional. Yet another example in Section 4 reveals that the dual to a GW process can be a rank-dependent GW process which is not a GW process with an eternal particle.

\section{Coupled rank-dependent GW processes and their duals}

Let $\Phi_{0}$ be the class of monotone functions $U: \mathbb{N}_{0} \rightarrow \mathbb{N}_{0}$ such that $U(0)=0$. If $U \in \Phi_{0}$ and $u(x)=U(x)-U(x-1)$, then we call $U$ a reproduction mapping with offspring numbers $u(x), x \in \mathbb{N}$. Given a set of random variables (1) which are mutually independent but not necessarily identically distributed, define a sequence of random reproduction mappings $U_{t}(x)=\sum_{y=1}^{x} u_{t}(y)$, and consider the family of iterations

$$
U_{a, b}=U_{b-1} \circ U_{b-2} \circ \cdots \circ U_{a} \quad \text { for } a<b, \quad U_{a, a}(x) \equiv x .
$$

Setting $Z_{t}=U_{a, t}\left(Z_{a}\right)$, we obtain a time-inhomogeneous Markov chain $\left\{Z_{t}\right\}_{t \geq a}$ which satisfies (2) and which we call a rank-dependent GW process. Moreover, using the system of stochastic iterations

$$
\boldsymbol{U}=\left\{U_{a, b}\right\}_{\infty<a \leq b<\infty},
$$

we can define coupled Markov chains $\left\{U_{a, t}(x)\right\}_{t \geq a}$ starting at $U_{a, a}(x)=x$ for all possible $a \in \mathbb{Z}$ and $x \in \mathbb{N}$ (cf. [7]). We call (3) a rank-dependent GW system with reproduction law

$$
f_{t, x}(s)=\mathbb{E}\left[s^{u_{t}(x)}\right], \quad s \in[0,1], x \in \mathbb{N}, t \in \mathbb{Z} .
$$

Definition 1. If $U \in \Phi_{0}$ and $V=U^{-}$, where

$$
U^{-}(x)=\min \{y: U(y) \geq x\},
$$

then $V \in \Phi_{0}$ is the pathwise dual of the reproduction mapping $U$. 
As shown in Section 5, Definition 1 is equivalent to the equality

$$
\left\{(x, y) \in \mathbb{N}_{0}^{2}: V(x) \leq y\right\}=\left\{(x, y) \in \mathbb{N}_{0}^{2}: U(y) \geq x\right\},
$$

and therefore it can be referred to as the pathwise Siegmund duality (see [13], [27], and [28]).

Definition 2. Given a rank-dependent GW system (3), define its time reverse by

$$
\boldsymbol{V}=\left\{V_{b, a}\right\}_{\infty<a \leq b<\infty}, \quad V_{b, a}=V_{a} \circ \cdots \circ V_{b-1} \text { for } a<b, V_{a, a}(x) \equiv x,
$$

where $V_{t}=U_{t}^{-}$are the dual reproduction mappings. Setting $\hat{U}_{t}=V_{-t-1}$, define the pathwise dual of $\boldsymbol{U}$ by

$$
\hat{\boldsymbol{U}}=\left\{\hat{U}_{a, b}\right\}_{\infty<a \leq b<\infty}, \quad \hat{U}_{a, b}=\hat{U}_{b-1} \circ \cdots \circ \hat{U}_{a} \text { for } a<b, \hat{U}_{a, a}(x) \equiv x .
$$

The trajectories of a rank-dependent GW system and its time reverse can be represented by forest graphs on the grid of nodes $\mathbb{N}_{0} \times \mathbb{Z}$. As shown in Figure 1(a), the bottom-up lineages $\left\{\left(U_{a, t}(x), t\right), t \geq a\right\}$ starting from different levels $a \in \mathbb{Z}$ and different positions $x \in \mathbb{N}_{0}$, merge into coalescent trees. We call the resulting graph a dual forest. On the other hand, as shown in Figure 1(b), the top-down lineages $\left\{\left(V_{b, t}(x), t\right), t \leq b\right\}$ starting from different levels $b \in \mathbb{Z}$ and different positions $x \in \mathbb{N}_{0}$, build up a graph that we call a primary forest. In Figure 1(c) we illustrate that the two forests can be conveniently depicted together after the dual forest is shifted to the right by $\frac{1}{2}$. Drawn this way, the lineages of the primary and dual forests do not cross. The primary forest describes the genealogical trees of the primary rank-dependent GW system. A lineage in the dual tree followed up from vertex $(z, t)$ delineates a trajectory of the Markov chain (2).

The remarkable fact that the dual forests do not cross is formally established as follows. If $x \rightarrow y$ is an edge of the dual forest connecting a pair of vertices at levels $t$ and $t+1$, then the two neighbour edges $y \rightarrow x_{1}$ and $y+1 \rightarrow x_{2}$ of the primary forest connecting levels $t+1$ and $t$ are such that $x_{1}<x+\frac{1}{2}<x_{2}$, and, therefore, no edge in the primary forest graph crosses the shifted edge $x+\frac{1}{2} \rightarrow y+\frac{1}{2}$. To verify the inequalities we have just asserted, observe that, by Definition 1, the relation $y=U_{t}(x)$ implies that $V_{t}(y)<x+\frac{1}{2}<V_{t}(y+1)$.

\section{Propositions 1-2 and Theorem 1}

Let the reproduction mappings $U, V, \tilde{U} \in \Phi_{0}$ be related by $V=U^{-}$and $\tilde{U}=V^{-}$. Use the same notation with the time index $t$.

Proposition 1. Given (3), let $V_{t}=U_{t}^{-}, \hat{U}_{t}=V_{-t-1}, \hat{V}_{t}=\hat{U}_{t}^{-}$, and $\tilde{U}_{t}=\hat{V}_{-t-1}$. Alongside the rank-dependent $G W$ system $\boldsymbol{U}$, consider its time reverse $\boldsymbol{V}$ and pathwise dual $\hat{\boldsymbol{U}}$ as in Definition 2. Whenever $a \leq b$ and $x, y \in \mathbb{N}_{0}$, the following two events coincide:

$$
\left\{\hat{U}_{-b,-a}(x) \leq y\right\}=\left\{x \leq U_{a, b}(y)\right\} .
$$

The reproduction mappings $\tilde{U}_{t}$ define a rank-dependent $G W$ system $\tilde{\boldsymbol{U}}$ in a similar way to the definition given in (3) of the primary rank-dependent $G W$ system $\boldsymbol{U} . \tilde{U}$ is the dual of the dual $\hat{\boldsymbol{U}}$, and can be obtained as a simple shifted transform of $\boldsymbol{U}$ :

$$
\tilde{U}_{a, b}(x)=U_{a, b}(x-1)+1, \quad x \geq 1, a \leq b .
$$




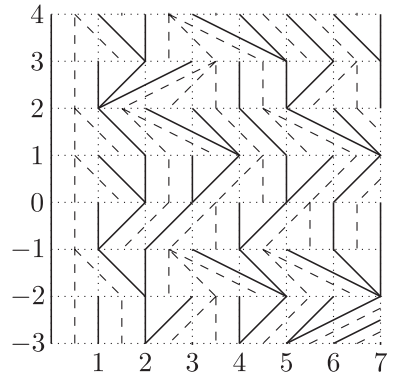

(a)

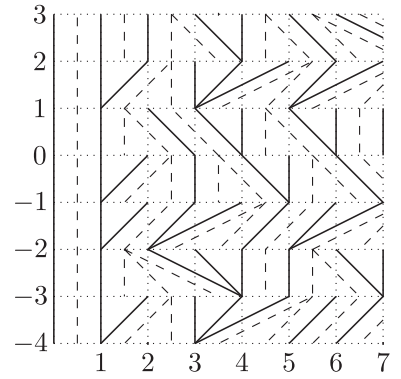

(b)

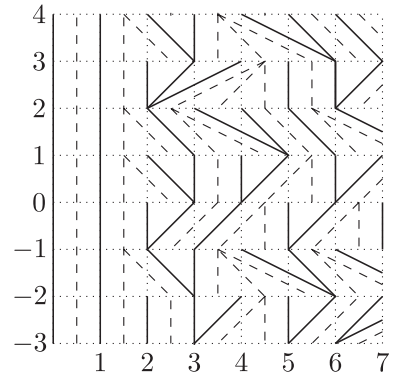

(c)

Figure 2: Graphical illustration of Proposition 1. The twofold dual in (c) is a shifted copy of the primary forest given in (a).

In Figure 2 we illustrate a diagrammatic proof of Proposition 1 using the graphical representation. In Figure 2(b) we show an intermediate step in the transformation of the primary forest in Figure 2(a) into the primary forest in Figure 2(c), representing the twofold dual rankdependent GW system $\tilde{\boldsymbol{U}}$. Since $\hat{U}_{a, b}=V_{-b,-a}$, we find that the primary forest in Figure 2(b), representing the genealogical trees of the dual rank-dependent GW system $\hat{\boldsymbol{U}}$, is the dual forest in Figure 2(a) flipped around the axis $t=0$ and shifted to the right by 1 (visually it is shifted by just $\frac{1}{2}$ ). Observe that the rank-1 particle in the dual reproduction flow is necessarily 'eternal', giving birth to at least one offspring. Thus, the unbroken-line forest from (a) flips into the broken-line forest in (b), then the unbroken-line forest in (b) generates the broken-line forest in (b), which in turn gives the unbroken-line forest in (c). We see that the primary forest in Figure 2(c) is a shifted copy of the primary forest in Figure 2(a), as claimed in Proposition 1.

The next result describes the case of a GW reproduction mapping $U$, that is, a system in which the corresponding offspring numbers $\{u(x), x \in \mathbb{N}\}$ are i.i.d.

Proposition 2. Let $V$ be the dual mapping of the reproduction mapping $U$ as in Definition 1 . Then the mapping $U$ generates the $G W$ reproduction law

$$
\mathbb{P}(u(x)=k)=P_{k}, \quad k \geq 0, x \geq 1,
$$

if and only if the dual offspring numbers have representation

$$
(v(1), v(2), \ldots)=(\xi_{1}+1, \underbrace{0, \ldots, 0}_{\eta_{1}}, \xi_{2}+1, \underbrace{0, \ldots, 0}_{\eta_{2}}, \ldots),
$$

where $\xi_{1}, \eta_{1}, \xi_{2}, \eta_{2}, \ldots$ are mutually independent $\mathbb{N}_{0}$-valued random variables with marginal distributions

$$
\mathbb{P}\left(\xi_{i}=k\right)=P_{0}^{k}\left(1-P_{0}\right), \quad \mathbb{P}\left(\eta_{i}=k\right)=\frac{P_{k+1}}{1-P_{0}}, \quad k \geq 0, i \geq 1 .
$$

In this case, the marginal dual reproduction law has the linear-fractional distribution

$$
\mathbb{E}\left[s^{v(x)}\right]=1-\hat{p}(x)+\hat{p}(x) \frac{P_{0} s}{1-\left(1-P_{0}\right) s},
$$

where $\hat{p}(1)=1$ and the sequence of parameters $\{\hat{p}(x), x \geq 2\}$ is defined by its generating 
function

$$
\sum_{x=1}^{\infty} \hat{p}(x+1) s^{x}=\frac{f(s)-f(0)}{1-f(s)}, \quad f(s)=\sum_{k=0}^{\infty} P_{k} s^{k} .
$$

A natural question arising in connection with Proposition 2 is whether it is possible for both the primary and its dual reproduction mappings to be GW? The answer is no, since the dual law always assigns at least one offspring to the particle of rank 1 . The closest to GW that the dual can be is a $G W$ reproduction with an eternal particle, which, by definition, is a rank-dependent GW reproduction mapping $V$ whose offspring numbers are such that $v(1) \geq 1$ and $v(2), v(3), \ldots$ have a common distribution.

The following result extends [18, Proposition 3.6] significantly.

Theorem 1. Let $U$ be a $G W$ reproduction mapping for which $\mathbb{E}\left[s^{u(x)}\right]=f(s)$ for all $x \geq 1$. Its pathwise dual $V$ is a $G W$ reproduction with an eternal particle if and only if

$$
f(s)=1-q+q \frac{p s}{1-(1-p) s}, \quad p, q \in(0,1] .
$$

Then

$$
\mathbb{E}\left[s^{v(x)}\right]= \begin{cases}\frac{q s}{1-(1-q) s} & \text { for } x=1, \\ 1-p+p \frac{q s}{1-(1-q) s} & \text { for } x=2,3, \ldots\end{cases}
$$

\section{Examples}

Example 1. (Pure-death rank-dependent GW.) A distinct forest structure appears in the case that the offspring numbers are $\{0,1\}$-valued, so

$$
f_{t, x}(s)=p_{t, x}+\left(1-p_{t, x}\right) s, \quad x \in \mathbb{N}, t \in \mathbb{Z} .
$$

Each dual lineage followed upwards eventually vanishes without branching. Given $p_{t, x} \equiv p_{x}$, the dual reproduction is not rank-dependent GW because of the dependence in the joint distribution

$$
\begin{aligned}
\mathbb{P}(v(1) & \left.=k_{1}, \ldots, v(m)=k_{m}\right) \\
& =p_{1} \ldots p_{k_{1}-1}\left(1-p_{k_{1}}\right) \prod_{l=1}^{m-1} p_{k_{1}+\cdots+k_{l}+1} \ldots p_{k_{1}+\cdots+k_{l+1}-1}\left(1-p_{k_{1}+\cdots+k_{l+1}}\right) .
\end{aligned}
$$

Example 2. (Birth-death $G W$ reproduction.) Consider a $G W$ reproduction law $\mathbb{P}(u=k)=p_{k}$ for which $p_{0}+p_{1}+p_{2}=1$. If $p_{2}=0$ then the dual reproduction is $\mathrm{GW}$ with the shifted geometric distribution

$$
\mathbb{P}(v(x)=k)=p_{0}^{k-1}\left(1-p_{0}\right), \quad k \geq 1 .
$$

If $p_{1}=0$ then the dual reproduction is rank-dependent $\mathrm{GW}$ described by the example given in the introduction, with $p=p_{0}$. On the other hand, if $p_{0}=0$ then the dual reproduction law is not rank-dependent $\mathrm{GW}$ because of the dependence

$$
\begin{array}{ll}
\mathbb{P}(v(1)=1)=1, & \mathbb{P}(v(2)=0, v(3)=1)=p_{2}, \\
\mathbb{P}(v(2)=1, v(3)=0)=p_{1} p_{2}, & \mathbb{P}(v(1)=0, v(3)=1)=p_{1}^{2} .
\end{array}
$$


Example 3. (Bounded GW processes.) Let the offspring probability generating functions of a rank-dependent GW process be given by

$$
f_{t, x}(s)=\left\{\begin{array}{ll}
f(s) & \text { for } x \in\left[1, B_{t}\right], \\
1 & \text { for } x>B_{t},
\end{array} \quad s \in[0,1], t \geq 0 .\right.
$$

This specifies a version of a truncated GW process with a stationary reproduction $f$, where the number of particles allowed to reproduce at time $t$ is bounded by $B_{t}$. Zubkov [30] obtained an interesting result for such processes in the supercritical case.

Example 4. ( $G W$ processes with immigration.) Specify a rank-dependent GW process via $f_{t, 1}(s)=s g_{t}(s)$ and $f_{t, x} \equiv f_{t}, x \geq 2$. This is a GW process with an eternal particle in a varying environment. Removing the eternal particle of rank 1 and keeping its offspring as immigrants yields a GW process with immigration. Such processes are well studied in the case of a stationary reproduction law $f_{t}=f$ and varying immigration $\left\{g_{t}\right\}_{t \geq 0}$ (see [22]). The case of varying $\left\{g_{t}, f_{t}\right\}_{t \geq 0}$ has received less attention in the literature (but see [20]).

Example 5. (GW processes with emigration.) Consider a time-homogeneous GW process with an eternal particle, such that (8) holds for $x \geq 2$, and $u(1) \geq 1$ has an arbitrary distribution. Its dual Markov chain can be interpreted in terms of a GW process with emigration (catastrophes, disasters), with a random number $\eta_{t} \stackrel{\mathrm{D}}{=} u(1)-1$ of particles being removed from generation $t$. If the current size $Y_{t}$ does not exceed $\eta_{t}$, the population dies out. Vatutin [29] was amongst the first to address this model, studying the critical case under the assumption that $\eta_{t} \equiv 1$.

Grey [9] showed that if the GW reproduction is supercritical and the numbers of emigrants $\left\{\eta_{t}\right\}_{t \geq 0}$ are independent copies of $\eta$, then the GW process with emigration goes extinct with probability 1 if and only if $\mathbb{E}[\log (\eta+1)]=\infty$. On the other hand, a well-known result in [1] is that a subcritical GW process with immigration has a stationary distribution if and only if $\mathbb{E}[\log (\eta+1)]<\infty$, where $\eta \stackrel{\mathrm{D}}{=} u(1)-1$ denotes the number of immigrants.

Example 6. (Rank-dependent $G W$ process with a carrying capacity.) Consider the timehomogeneous case, $f_{t, x}=f_{x}$, in which the reproduction law is variable along the spatial position. Our setting is suitable for modelling population size-dependent reproduction in a way which is different from that of [17] and [19]. Let $m_{x}=f_{x}^{\prime}(1)$ be the mean offspring number for the particle of rank $x$. Suppose that $m_{1}>1$, that $m_{x}$ decreases monotonically with $x$, and that, for some $K \in \mathbb{N}$,

$$
m_{1}+\cdots+m_{x} \geq x, \quad x \leq K, \quad m_{1}+\cdots+m_{x}<x, \quad x>K .
$$

Then $K$ can be viewed as the carrying capacity of a population of individuals producing on average fewer than 1 child per individual when the size of the population exceeds $K$.

Example 7. (Embeddable rank-dependent GW processes.) Embeddability of basic GW processes into continuous-time Markov branching processes is not a fully resolved issue [3, Chapter III.12]. Several explicit examples of embeddable GW processes can be found in [24]. One known class of embeddable GW processes in varying environments is the case of linearfractional reproduction addressed in Theorem 1.

Consider a continuous-time linear birth-death process $\left\{Z(t), t \geq t_{0}\right\}$ with variable birth and death rates $\{\lambda(t), \mu(t)\}_{t \in \mathbb{R}}$ per individual. It is well known that such a process has linearfractional distributions. By [14], with $\rho\left(t_{0}, t\right)=\int_{t_{0}}^{t}[\mu(u)-\lambda(u)] \mathrm{d} u$,

$$
\mathrm{E}\left[s^{Z(t)} \mid Z\left(t_{0}\right)=1\right]=1-q\left(t_{0}, t\right)+q\left(t_{0}, t\right) \frac{p\left(t_{0}, t\right) s}{1-\left(1-p\left(t_{0}, t\right)\right) s},
$$




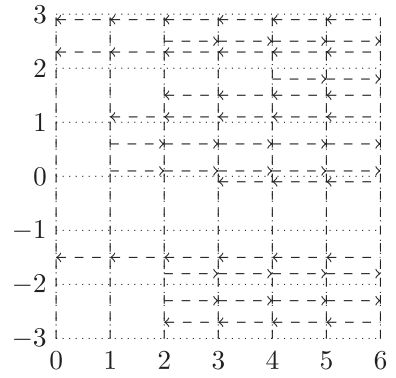

(a)

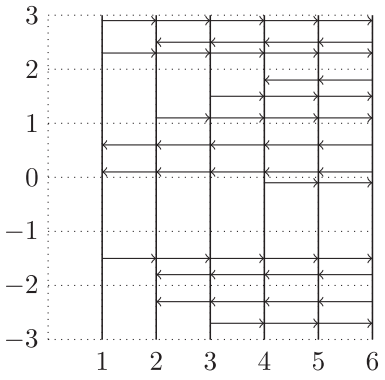

(b)

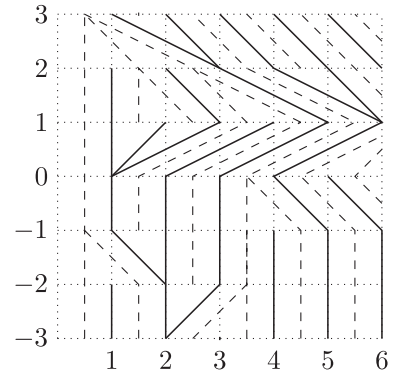

(c)

FiguRE 3: Graphical representation of birth-death processes. Arrows to the left mean the death at the end position of the line of arrows. Arrows to the right mean splitting at the origin of the line of arrows.

where

$$
q\left(t_{0}, t\right)=\frac{1}{1+\int_{t_{0}}^{t} \mathrm{e}^{\rho\left(t_{0}, u\right)} \mu(u) \mathrm{d} u}, \quad p\left(t_{0}, t\right)=\mathrm{e}^{\rho\left(t_{0}, t\right)} q\left(t_{0}, t\right) .
$$

A linear-fractional GW process with varying parameters $\left(q_{t}, p_{t}\right)$ in $(8)$ has

$$
q_{t}=\frac{1}{1+\int_{t-1}^{t} \mathrm{e}^{\rho_{u}} \mu(u) \mathrm{d} u}, \quad p_{t}=\mathrm{e}^{\rho_{t}} q_{t}, \quad \rho_{u}:=\rho(t-1, u)=\int_{t-1}^{u}[\mu(v)-\lambda(v)] \mathrm{d} v,
$$

and can be embedded in a birth-death process. In Figure 3 we illustrate the graphical representation for such an embedding. See also a recent result [8] presenting a different approach to dual random forests in a continuous-time setting.

Example 8. (Defective rank-dependent $G W$.) For any $V \in \Phi_{0}$, the $\operatorname{limit} \bar{V}=\lim _{x \rightarrow \infty} V(x)$ is either finite or infinite. We call a random reproduction mapping $U \in \Phi_{0}$ defective if its dual $V$ satisfies $\mathbb{P}(\bar{V}<\infty)>0$. In the defective case, a particle can produce infinitely many offspring. GW processes with a defective reproduction law were studied in a recent paper [25].

Turning to nonlinear birth-death processes (see, e.g. [26]), observe that, in general, the embedding discussed in the previous example does not yield a rank-dependent GW process, because the numbers of offspring may depend on each other. An interesting exception is the pure-death processes producing the embedded pure-death rank-dependent GW processes mentioned in Example 1. Let the time-homogeneous death rates $\mu_{x}$ depend on the ranks $x \geq 1$ of individuals and be such that $\sum_{x=1}^{\infty}\left(\mu_{1}+\cdots+\mu_{x}\right)^{-1}<\infty$; the result is a pure-death process coming down from infinity (see, e.g. [23]). Observe that in this case the dual Markov chain gives a defective reproduction model which is not a rank-dependent GW process.

\section{Proofs of Propositions 1-2 and Theorem 1}

Let reproduction mappings $U, V, \tilde{U} \in \Phi_{0}$ be related via $V=U^{-}$and $\tilde{U}=V^{-}$. Denote the corresponding offspring numbers by $u(x), v(x)$, and $\tilde{u}(x)$.

Lemma 1. Define $\left(\xi_{i}, \eta_{i}\right)$ by

$$
(u(1), u(2), \ldots)=(\underbrace{0, \ldots, 0}_{\xi_{1}}, \eta_{1}+1, \underbrace{0, \ldots, 0}_{\xi_{2}}, \eta_{2}+1, \ldots), \quad \xi_{i}, \eta_{i} \geq 0, i \in \mathbb{N} .
$$


Then (5) holds and

$$
(\tilde{u}(1), \tilde{u}(2), \ldots)=(1, \underbrace{0, \ldots, 0}_{\xi_{1}}, \eta_{1}+1, \underbrace{0, \ldots, 0}_{\xi_{2}}, \eta_{2}+1, \ldots) .
$$

Proof. From $V(x)=\min \{y: U(y) \geq x\}$, it follows that $V(0)=0$ and

$$
\{x: V(x)=y\}=\{x: U(y-1)<x \leq U(y)\}, \quad y \geq 1,
$$

which implies (5). Similarly, (11) follows from (5). Observe also that (12) entails (4).

Proof of Proposition 1. Using (4), we obtain consecutively

$$
\begin{aligned}
\left\{\hat{U}_{-b,-a}(x) \leq y\right\} & =\left\{\hat{U}_{-a-1} \circ \cdots \circ \hat{U}_{-b}(x) \leq y\right\} \\
& =\left\{V_{a} \circ \cdots \circ V_{b-1}(x) \leq y\right\} \\
& =\left\{V_{a+1} \circ \cdots \circ V_{b-1}(x) \leq U_{a}(y)\right\} \\
& \vdots \\
& =\left\{x \leq U_{b-1} \circ \cdots \circ U_{a}(y)\right\} \\
& =\left\{x \leq U_{a, b}(y)\right\} .
\end{aligned}
$$

Observe that $\tilde{U}_{t}=V_{t}^{-}$, and, by Lemma $1, \tilde{U}_{t}(x)=U_{t}(x-1)+1$, which yields

$$
\begin{aligned}
\tilde{U}_{a, b}(x) & =\tilde{U}_{b-1} \circ \cdots \circ \tilde{U}_{a}(x) \\
& =\tilde{U}_{b-1} \circ \cdots \circ \tilde{U}_{a+1}\left(U_{a}(x-1)+1\right) \\
& =\tilde{U}_{b-1} \circ \cdots \circ \tilde{U}_{a+2}\left(U_{a+1} \circ U_{a}(x-1)+1\right) \\
& =U_{a, b}(x-1)+1 .
\end{aligned}
$$

Proof of Proposition 2. The random variables $u(1), u(2), \ldots$ are independent with common distribution $\left\{P_{k}\right\}_{k=0}^{\infty}$ if and only if (10) holds with mutually independent $\xi_{1}, \eta_{1}, \xi_{2}, \eta_{2}, \ldots$ such that, for $k \geq 0$ and $i \geq 1$,

$$
\mathbb{P}\left(\xi_{i}=k\right)=P_{0}^{k}\left(1-P_{0}\right), \quad \mathbb{P}\left(\eta_{i}=k\right)=\mathbb{P}(u(1)=k+1 \mid u(1) \geq 1), \quad k \geq 0, i \geq 1 .
$$

By Lemma 1, this proves the first statement of the proposition.

Turning to the second statement concerning the distribution of $v(x)$, let $\hat{p}(x)=\mathbb{P}(v(x)>0)$. The first statement implies that (6) holds, and, for $x \geq 1$,

$$
\hat{p}(x)=\mathbb{P}\left(\bigcup_{n=0}^{\infty}\left\{1+n+\eta_{1}+\cdots+\eta_{n}=x\right\}\right)=\sum_{n=0}^{x-1} \mathbb{P}\left(\eta_{1}+\cdots+\eta_{n}=x-n-1\right) .
$$

This implies that $\hat{p}(1)=1$, and, for $x \geq 2$, using conditioning on $\eta_{1}$, we derive the recursion

$$
\begin{aligned}
\hat{p}(x) & =\sum_{n=1}^{x-1} \mathbb{P}\left(\eta_{1}+\cdots+\eta_{n}=x-n-1\right) \\
& =\sum_{n=1}^{x-1} \sum_{k=1}^{x-n} \mathbb{P}\left(\eta_{1}=k-1\right) \mathbb{P}\left(\eta_{2}+\cdots+\eta_{n}=x-n-k\right) \\
& =\left(1-P_{0}\right)^{-1} \sum_{k=1}^{x-1} P_{k} \sum_{n=1}^{x-k} \mathbb{P}\left(\eta_{2}+\cdots+\eta_{n}=x-k-n\right) \\
& =\left(1-P_{0}\right)^{-1} \sum_{k=1}^{x-1} P_{k} \hat{p}(x-k) .
\end{aligned}
$$


In terms of the generating function $Q(s)=\sum_{x=1}^{\infty} \hat{p}(x+1) s^{x}$, this recursion gives

$$
Q(s)=\frac{f(s)-P_{0}}{1-P_{0}}(1+Q(s))
$$

which implies (7), as asserted.

Proof of Theorem 1. In view of Proposition 2, the pathwise dual $V$ is a GW reproduction with an eternal particle if and only if $\hat{p}(x)=p$ for all $x \geq 2$. In this case, (6) implies (9) with $q=f(0)$. On the other hand, (7) takes the form

$$
\frac{f(s)-q}{1-f(s)}=\sum_{x=1}^{\infty} p s^{x}=\frac{p s}{1-s},
$$

which yields (8).

\section{Acknowledgements}

The authors thank Uwe Rösler for a discussion of an issue concerning duality, as well as an anonymous referee for valuable comments. The research by Jonas Jagers was supported by the Royal Swedish Academy of Sciences through the Elis Sidenbladh foundation grant.

\section{References}

[1] Asmussen, S. and Hering, H. (1983). Branching Processes. Birkhäuser, Boston, MA.

[2] Asmussen, S. and Sigman, K. (1996). Monotone stochastic recursions and their duals. Prob. Eng. Inf. Sci. 10, $1-20$.

[3] Athreya, K. B. And Ney, P. E. (1972). Branching Processes. Springer, New York.

[4] Bansaye, V. And Simatos, F. (2015). On the scaling limits of Galton-Watson processes in varying environments. Electron. J. Prob. 20, 36pp.

[5] Bertoin, J. (2009). The structure of the allelic partition of the total population for Galton-Watson processes with neutral mutations. Ann. Prob. 37, 1502-1523.

[6] Braunsteins, P. and Hautphenne, S. (2017). Extinction in lower Hessenberg branching processes with countably many types. Preprint. Available at https://arxiv.org/abs/1706.02919v1.

[7] Diaconis, P. and Freedman, D. (1999). Iterated random functions. SIAM Rev. 41, 45-76.

[8] Felipe, M. D. and Lambert, A. (2015). Time reversal dualities for some random forests. ALEA 12, 399-426.

[9] Grey, D. R. (1988). Supercritical branching processes with density independent catastrophes. Math. Proc. Camb. Phil. Soc. 104, 413-416.

[10] Grosjean, N. And Huillet, T. (2016). On a coalescence process and its branching genealogy. J. Appl. Prob. 53, 1156-1165.

[11] Haccou, P., Jagers, P. and Vatutin, V. A. (2005). Branching Processes: Variation, Growth and Extinction of Populations. Cambridge University Press.

[12] Jagers, P. (1974). Galton-Watson processes in varying environments. J. Appl. Prob. 11, 174-178.

[13] Jansen, S. And Kurt, N. (2014). On the notion(s) of duality for Markov processes. Prob. Surveys 11, 59-120.

[14] Kendall, D. G. (1948). On the generalized 'birth-and-death' process. Ann. Math. Statist. 19, 1-15.

[15] Kersting, G. (2017). A unifying approach to branching processes in varying environments. Preprint. Available at https://arxiv.org/abs/1703.01960v6.

[16] Kimmel, M. And Axelrod, D. E. (2015). Branching Processes in Biology, 2nd edn. Springer, New York.

[17] Klebaner, F. C. (1984). On population-size-dependent branching processes. Adv. Appl. Prob. 16, 30-55.

[18] Klebaner, F. C., Rösler, U. and Sagitov, S. (2007). Transformations of Galton-Watson processes and linear fractional reproduction. Adv. Appl. Prob. 39, 1036-1053.

[19] Klebaner, F. C. et al. (2011). Stochasticity in the adaptive dynamics of evolution: the bare bones. J. Biol. Dynam. 5, 147-162.

[20] Mitov, K. V. AND OMey, E. (2014). A branching process with immigration in varying environments. Commun. Statist. Theory Meth. 43, 5211-5225.

[21] MöHle, M. (1999). The concept of duality and applications to Markov processes arising in neutral population genetics models. Bernoulli 5, 761-777. 
[22] Rahimov, I. (1995). Random Sums and Branching Stochastic Processes. Springer, New York.

[23] Sagitov, S. and France, T. (2017). Limit theorems for pure death processes coming down from infinity. J. Appl. Prob. 54, 720-731.

[24] Sagitov, S. ANd Lindo, A. (2016). A special family of Galton-Watson processes with explosions. In Branching Processes and Their Applications (Lecture Notes Statist. 219), Springer, pp. 237-254.

[25] Sagitov, S. and Minuesa, C. (2017). Defective Galton-Watson processes. Stoch. Models 33, 451-472.

[26] Sagitov, S. and Shaimerdenova, A. (2013). Extinction times for a birth-death process with weak competition. Lithuanian Math. J. 53, 220-234.

[27] Siegmund, D. (1976). The equivalence of absorbing and reflecting barrier problems for stochastically monotone Markov processes. Ann. Prob. 4, 914-924.

[28] Sturm, A. and Swart, J. M. (2018). Pathwise duals of monotone and additive Markov processes. J. Theoret. Prob. 31, 932-983.

[29] Vatutin, V. A. (1977). A critical Galton-Watson branching process with emigration. Theory Prob. Appl. 22, $465-481$.

[30] Zubkov, A. M. (1970). A condition for the extinction of a bounded branching process. Math. Notes 8, $472-477$.

\section{SERIK SAGITOV, Chalmers University of Technology and University of Gothenburg}

Department of Mathematical Sciences, Chalmers University of Technology, 41296 Gothenburg, Sweden.

Email address: serik@chalmers.se

JONAS JAGERS, Chalmers University of Technology

Department of Mathematical Sciences, Chalmers University of Technology, 41296 Gothenburg, Sweden.

Email address: jagersj@student.chalmers.se 